\title{
Light curves and absolute magnitudes of four recent fast LMC novae
}

\author{
J.B. Hearnshaw, C.M. Livingston, A.C. Gilmore, P.M. Kilmartin \\ Dept of Physics and Astronomy and Mt John University Observatory, \\ University of Canterbury, Private Bag 4800, Christchurch, New Zealand
}

\begin{abstract}
The light curves of four recent fast LMC novae (Nova LMC 1988a, 1992, 1995, 2000) have been analysed to obtain the parameter $t_{2}$, the time for a two magnitude decline below maximum light. Using the calibration of Della Valle \& Livio (1995), values of $M_{V}$ at maximum are obtained. The weighted mean distance modulus to the LMC based on these novae is $18.89 \pm 0.16$. This differs significantly from the distance modulus adopted by Della Valle \& Livio of 18.50 , but only differs at the $1 \sigma$-level from Feast's (1999) value of $18.70 \pm 0.10$. The evidence based on these novae suggests that either: (i) $\mathrm{DM}_{\mathrm{LMC}}=18.50$ is too close for the LMC; or (ii) some novae in the LMC, including these four, are significantly underluminous at maximum light compared with those in M31, by about $0.4 \mathrm{mag}$. This could be a metallicity effect, given that more metal-rich M31 novae were predominantly used by Della Valle \& Livio to obtain their calibration.
\end{abstract}

\section{Observations and analysis of light curves}

The observational data used were: (i) photoelectric $U B V(R I)_{\mathrm{C}}$ photometry obtained on a 61-cm telescope at Mt John University Observatory; supplemented by (ii) visual magnitude estimates from the AAVSO database (courtesy J.A. Mattei, 2001); (iii) visual magnitude estimates from the Variable Star Section of the Royal Astronomical Society of New Zealand (courtesy F.M. Bateson); and (iv) miscellaneous estimated or photometric (mainly CCD) magnitudes reported in IAU Circulars. The CCD data used a variety of different filters and were often not in the $V$-band. Figs 1 to 4 show the light curves.

The best $V$ light curves were drawn through the data points by eye and the parameters $t_{0}$ (time of maximum light) and $t_{2}$ (time interval for a 2 mag decline) were estimated. We also obtained $m_{V}(\max ), t_{3}$ (time for 3 mag decline) and $m_{15}$ (apparent magnitude $15 \mathrm{~d}$ post-maximum), together with best estimates of the error bars. Values are given in Table 1 . All of these novae may be classified as very fast $\left(t_{2}<10 \mathrm{~d}\right)$, or fast $\left(11 \mathrm{~d}<t_{2}<25 \mathrm{~d}\right)$ (Payne-Gaposchkin 1957).

\section{Calibration}

The $t_{2}$ parameters were converted to $M_{V}$ at maximum light using the non-linear calibration of Della Valle \& Livio (1995) based mainly on observations of 85 M31 


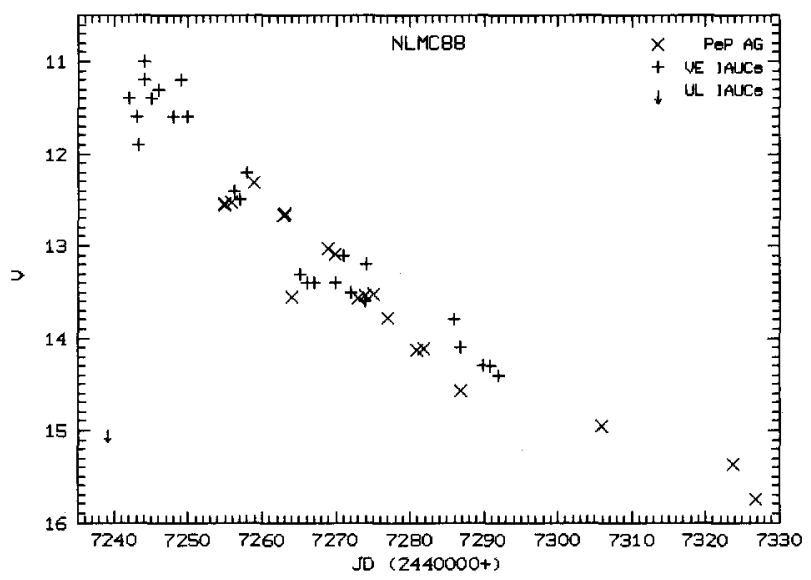

Figure 1. Light curve of Nova LMC1988.

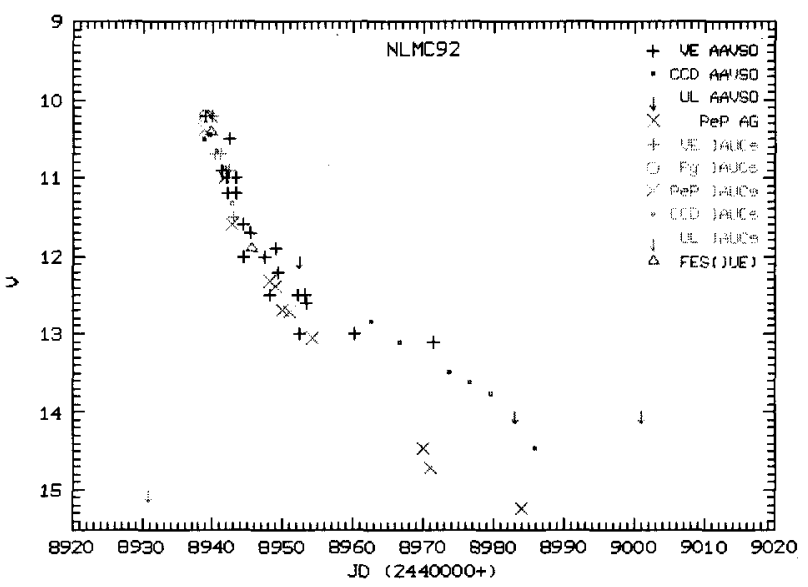

Figure 2. Light curve of Nova LMC1992.

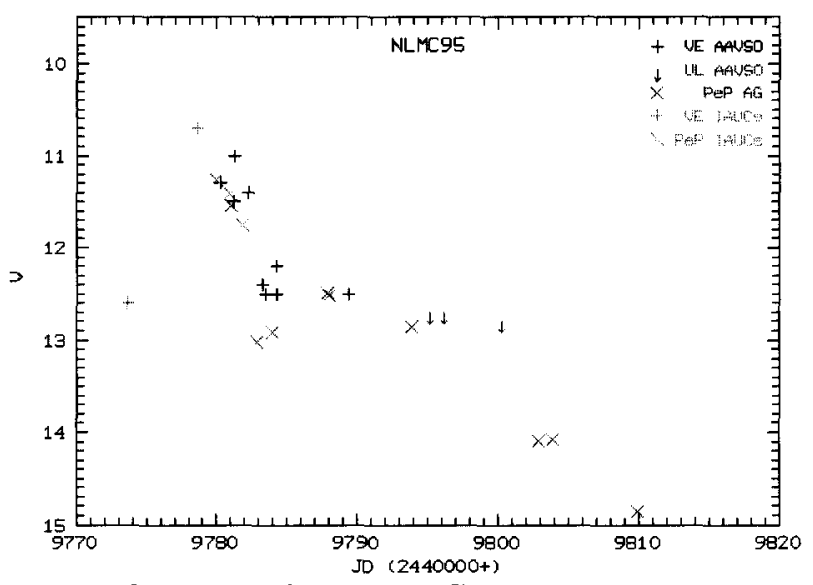

Figure 3. Light curve of Nova LMC1995. 


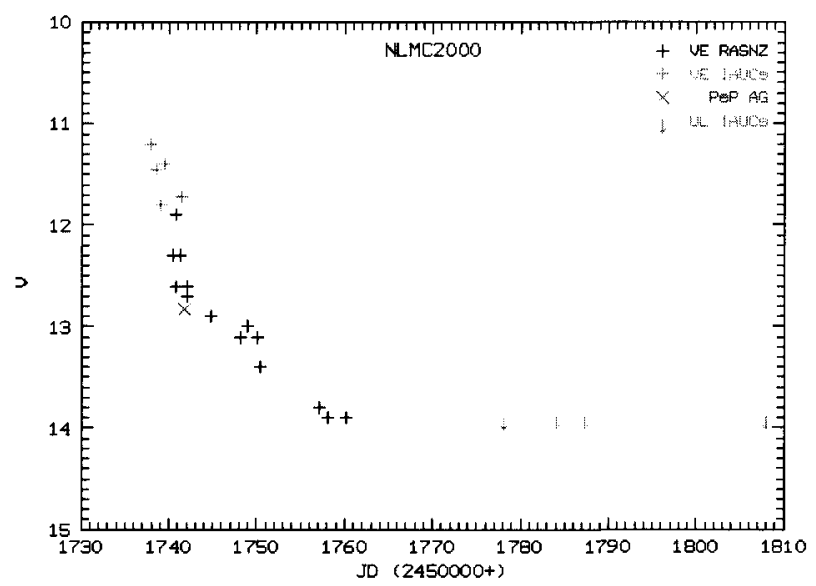

Figure 4. Light curve of Nova LMC2000.

novae with a distance modulus (DM) taken to be 24.30. A few (15) LMC novae $(\mathrm{DM}=18.50)$ were also used. Their calibration was

$$
M_{V}=-7.92-0.81 \arctan \left(\left(1.32-\log t_{2}\right) / 0.23\right) .
$$

Fig. 5 shows the novae and the best calibration curve as well as the curve at $3 \sigma$ from the mean. Triangles are calibrating LMC novae, while crosses are the four LMC novae of this analysis. It is notable that three of the calibrating LMC novae and one of the four novae analysed here lies about $5 \sigma$ below the mean curve. A further two LMC novae considered here are $3 \sigma$ below and one is $2 \sigma$ below.

Table 1. Novae parameters from visual light curves.

\begin{tabular}{lccccc}
\hline Nova & $t_{0}(\mathrm{JD})$ & $t_{2}(\mathrm{~d})$ & $t_{3}(\mathrm{~d})$ & $m_{V}(\max )$ & $m_{15}$ \\
\hline LMC1988a & $47245.5 \pm 2.0$ & $22.5 \pm 4.0$ & $38.4_{-5.0}^{+5.9}$ & $11.2 \pm 0.3$ & $12.62 \pm 0.18$ \\
LMC1992 & $48939.25 \pm 0.25$ & $6.9 \pm 1.1$ & $13.7 \pm 1.6$ & $10.0 \pm 0.2$ & $13.17 \pm 0.04$ \\
LMC1995 & $49777.0 \pm 0.8$ & $11.0 \pm 3.0$ & $19.6 \pm 3.2$ & $10.35 \pm 0.5$ & $12.83 \pm 0.08$ \\
LMC2000 & $51736.0 \pm 1.0$ & $8.0_{-3.5}^{+4.5}$ & $20.0_{-7.0}^{+12.0}$ & $10.7 \pm 0.5$ & $13.42 \pm 0.07$ \\
\hline \hline
\end{tabular}

Table 2. Absolute magnitudes and distance moduli of LMC novae.

\begin{tabular}{lccl}
\hline Nova & $M_{V}(\max )$ & DM & $\mathrm{d}(\mathrm{kpc})$ \\
\hline LMC1988a & $7.81_{-0.29}^{+0.23}$ & 18.96 & $61.9 \pm 11.6$ \\
LMC1992 & $8.83_{-0.05}^{+0.04}$ & 18.78 & $57.0 \pm 5.4$ \\
LMC1995 & $8.63_{-0.15}^{+0.19}$ & 18.93 & $61.1 \pm 11.0$ \\
LMC2000 & $8.78_{-0.14}^{+0.24}$ & 19.43 & $76.9 \pm 19.0$ \\
\hline
\end{tabular}




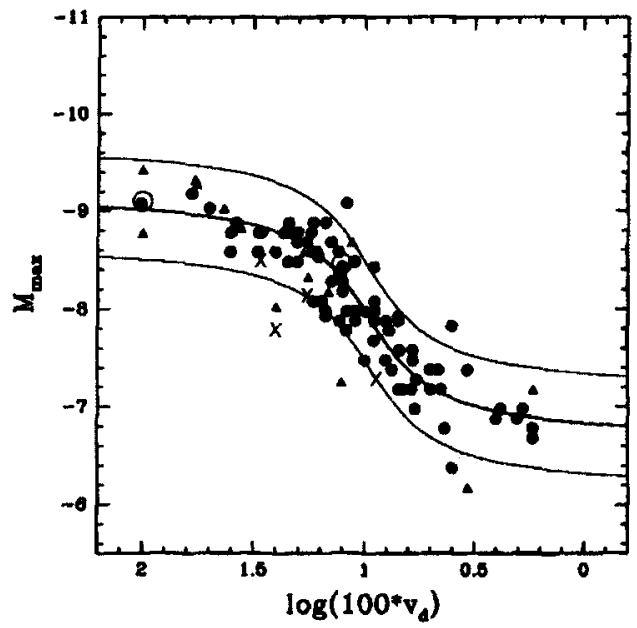

Figure 5. Maximum magnitude vs rate of decline calibration of Della Valle \& Livio (1995) showing M31 novae ( $\bullet$ ) and LMC calibration novae $(\triangle)$. LMC novae in this analysis are shown as $(x)$. The upper and lower curves are $3 \sigma(\sim 0.5 \mathrm{mag})$ from the mean.

\section{Distance moduli}

The distance moduli of the four LMC novae were obtained from the apparent and absolute magnitudes at maximum light, after taking an adopted extinction of $A_{V}=0.05$ mag into account. Results are shown in Table 2. The weighted mean distance modulus of these novae was $18.89 \pm 0.16$, corresponding to $60.0 \mathrm{kpc}$. The recent best value by Feast (1999) was $18.70 \pm 0.10(55.0 \mathrm{kpc})$ based on Cepheids, Mira stars, RR Lyrae stars, clump red giants and SN1987A, but not novae.

The data suggest that if $\mathrm{DM}_{\mathrm{LMC}}=18.50$, then these LMC novae, as well as several used by Della Valle \& Livio (1995), are on average 0.4 mag less bright at maximum than novae in M31 and those in the galaxy. That difference could be a fundamental difference, possibly caused by the lower metallicity of LMC stars. However the discrepancy becomes only $0.19 \pm 0.19 \mathrm{mag}$ if $\mathrm{DM}_{\mathrm{LMC}}=18.70$ is adopted. The simplest interpretation is therefore that LMC novae support the larger distance modulus of at least 18.70 for the LMC.

\section{References}

Della Valle, M., Livio, M. 1995, ApJ, 452, 704

Feast, M.W. 1999, in New Views of the Magellanic Clouds, eds. Y.-H. Chu, N. Suntzeff, J. Hesser, \& D. Bohlender, IAU Symp. 190, 542

Payne-Gaposchkin, C. 1957, The Galactic Novae, (North Holland Publishing Co., Amsterdam) 